\title{
PROJETO DE EXTENSÃO - RADIOLOGIA E COMUNIDADE: AVALIAÇÃO DAS ATIVIDADES DE PROMOÇÃO DE SAÚdE NA COMUNIDADE
}

\author{
Caroline de Medeiros \\ Instituto Federal de Santa Catarina \\ carol@ifsc.edu.br
}

Patricia Fernanda Dorow

Instituto Federal de Santa Catarina

patriciad@ifsc.edu.br

Juliana Almeida Coelho de Melo

Instituto Federal de Santa Catarina

julianac@ifsc.edu.br

Charlene da Silva

Instituto Federal de Santa Catarina

lene__@hotmail.com

\section{Resumo}

Conhecer as formas de prevenção e tratamento é primordial para lidar com patologias que acometem a saúde da mulher, como a osteoporose e o câncer de mama. O intercâmbio de conhecimentos entre instituições de ensino superior e sociedade facilita esse processo. Dessa forma, o objetivo geral do artigo foi avaliar a percepção dos participantes sobre ações de extensão universitária em saúde. Foram comparadas as avaliações feitas pelas três comunidades: Santinho, Costeira e Garopaba, localizadas na grande Florianópolis. Foi um estudo quantitativo, exploratório e descritivo que foi realizado no ano de 2013. Envolveu 170 participantes, sendo 14 discentes, 154 pessoas participantes da atividade e 2 docentes do Instituto Federal de Ciência e Tecnologia de Santa Catarina (IFSC). Como resultados foi possível verificar que a maioria da população acometida pela osteoporose e pelo câncer de mama eram de mulheres idosas. A avaliação do evento foi considerada de boa a ótima. Concluiu-se, que a interação entre estudantes e comunidades para a educação em saúde é uma ótima forma para os estudantes transmitirem seus conhecimentos para a comunidade e aprenderem com ela, formando uma espiral virtuosa de conhecimento explícito e tácito.

\section{(†) (-) Esta obra está licenciada sob uma Licença Creative Commons.}


Palavras-chave: Saúde da mulher; Educação permanente; Extensão universitária.

\title{
PROPOSED EXTENSION - RADIOLOGY AND COMMUNITY: ASSESSMENT OF ACTIVITIES FOR THE PROMOTION OF HEALTH IN THE COMMUNITY
}

\begin{abstract}
Knowing the ways of prevention and treatment is essential to deal with pathologies that affect women's health, such as osteoporosis and breast cancer. The exchange of knowledge between higher education institutions and society facilitates this process. Thus, the overall aim of the paper was to assess the participants' perception of the actions of university extension in health. Santinho, Coastal and Garopaba, located in the city of Florianópolis, the assessments made by the three communities were compared. Was a quantitative, exploratory and descriptive study was conducted in 2013. It involved 170 participants, including 14 students, 154 people participating in the activity and 2 teachers of Federal Institute of Science and Technology of Santa Catarina (IFSC). As a result we found that the majority of the population affected by osteoporosis and breast cancer were the elderly women. The evaluation of the event was considered good to excellent. It was concluded that the interaction between students and communities to health education is a great way for students to convey their knowledge to the community and learn from it, forming a virtuous spiral of explicit and tacit knowledge.
\end{abstract}

Keywords: Women's health, Continuing education, University extension. 
Projeto de extensão - radiologia e comunidade: avaliação das atividades de promoção de saúde na comunidade

\section{INTRODUÇÃO}

As Instituições de Ensino Superior respondem ativamente pelo crescimento sustentável e econômico do país, e essas devem ter estratégias que utilizem o conhecimento para estimular a inovação e melhorar os serviços educacionais. Salmi (2012) acredita que o setor de ensino superior deve desempenhar um papel central na preparação da sociedade para novos tempos. Da mesma forma, Loh, Grabosky e Basskuk (2003) apontam que os Institutos Federais de Educação possuem a responsabilidade social de implementar ações que possibilitem um efetivo compartilhamento do conhecimento além de suas fronteiras.

Esse projeto de extensão do Instituto Federal de Santa Catarina - IFSC é voltado aos discentes e para as comunidades as quais fazem parte do projeto. Está vinculado ao Curso Superior de Tecnologia em Radiologia e oferece atividades na área da saúde da mulher. Representa o compromisso do IFSC com a comunidade, assim como um mecanismo integrado ao processo de ensino e aprendizagem, oportunizando aos estudantes vivenciarem os conhecimentos aprendidos em sala de aula.

Dessa forma, o projeto contribuiu para a qualidade de vida durante o envelhecimento. Pois este constitui um fato com implicações médicas, econômicas, sociais e políticas. No processo de envelhecimento, o idoso sofre modificações biológicas, fisiológicas, cognitivas, patológicas e socioeconômicas necessitando, portanto, de atenção especial. No entanto, as particularidades da idade não podem determinar que o idoso se tornasse um ser doente e sim que tais modificações possam ser adaptáveis a uma vida ativa e saudável. Dessa forma, tornase essencial que os profissionais de saúde tomem consciência dos fatores determinantes desse processo, compreendendo sua complexidade e magnitude e atuando em prol da promoção da saúde desses idosos (MARTINS et al., 2007).

Com intuito de levar às comunidades carentes informações sobre duas das doenças que mais atingem a população brasileira, o câncer de mama e a osteoporose, o Curso Superior de Tecnologia - CST em Radiologia do Instituto Federal de Santa Catarina - IFSC, campus Florianópolis, possui em sua unidade curricular "Densitometria Óssea e Mamografia", a oferta anual de palestras sobre estes assuntos a comunidade local. Nesta unidade curricular, os estudantes reconhecem a importância desses exames diagnósticos para prevenir e detectar precocemente a osteoporose e o câncer de mama. Assim, o projeto integra os universitários e um setor específico da sociedade, as mulheres.

\section{Esta obra está licenciada sob uma Licença Creative Commons.}


Projeto de extensão - radiologia e comunidade: avaliação das atividades de promoção de saúde na comunidade

O Projeto de Extensão "Radiologia e Comunidade" foi idealizado pela docente Caroline de Medeiros no ano de 2006 e, no ano seguinte, contou com a contribuição da docente Juliana Almeida Coelho de Melo. O projeto vem colaborando na democratização do saber, na transmissão do conhecimento, e na construção compartilhada de novos saberes, promovendo assim o ensino em saúde.

Anualmente o desenvolvimento do projeto fica a cargo da turma de Radiologia que está no quinto módulo da graduação, cursando a disciplina Mamografia/Densitometria Óssea. Dessa forma, desde seu início o projeto já beneficiou centenas de mulheres, em diferentes comunidades da região da Grande Florianópolis.

Esse artigo apresenta de um relato de experiência que foi vivenciada por estudantes e docentes do CST em Radiologia do IFSC sobre a proposta interdisciplinar de inserir em uma unidade curricular de cunho técnico um projeto que prevê a aproximação dos estudantes com comunidades carentes de informação.

De acordo com Paviani (2003), a interdisciplinaridade pode se expressar por diferentes sujeitos voltados para um problema de uma única disciplina e ainda, pela aplicação de conhecimentos no campo de atuação profissional. Nessa perspectiva, o projeto "Radiologia e Comunidade" objetiva a aproximação do estudante ao seu campo de trabalho, bem como propiciar às comunidades carentes de informação esclarecimentos acerca do câncer de mama e osteoporose.

Além disso, esse projeto de extensão destaca os aspectos relacionados ao currículo integrado, pois se entende que essa é uma possibilidade de concretizar práticas educativas que permitam que estudante perceba a realidade para além do aparente, compreendendo o sentido da totalidade do objeto de estudo.

Com a realização de ações de extensão envolvendo a interdisciplinaridade do Curso Superior Tecnológico em Radiologia, acredita-se que as respostas necessárias a este projeto foram encontradas em caminhos que "reinventam" a própria formação científica e, principalmente pela condução interdisciplinar do grupo. Assim, ao compartilhar ações dividindo saberes com as atividades interdisciplinares, foi possível reconhecer que o cuidado a aspectos físicos e sociais, supera um modelo de aprendizagem fragmentada e garantiu aos envolvidos no projeto uma aprendizagem a qual reconheceu os sujeitos na sua totalidade (KAMINSKI, 2000).

\section{Esta obra está licenciada sob uma Licença Creative Commons.}


Projeto de extensão - radiologia e comunidade: avaliação das atividades de promoção de saúde na comunidade

Para Castro (2004), a maioria dos projetos de extensão se apresenta como a possibilidade da universidade interagir com a população e por consequência a possibilidade, parece que única, dos estudantes terem contato com o mundo fora da universidade. Portanto, mostra-se a importância deste elo entre comunidade e universidade como instrumento de transformação social por meio da educação em saúde.

A prática educativa em saúde se baseia em elementos que explicam o processo saúde/doença como as condições objetivas e subjetivas da existência, os direitos sociais, cuja conquista e usufruto dependem do conhecimento e da consciência deles e de como acessá-los. Esta consciência e conhecimento podem viabilizar o controle social, reconhecido como indispensável e característico do exercício cidadão (MOREIRA e PELLIZZARO, 2009).

Diante do exposto, este artigo apresenta a avaliação da percepção dos participantes sobre um evento de extensão, comparando a avaliação feita pelas três comunidades atendida: Santinho, Costeira e Garopaba. O projeto permitiu uma reflexão de temas como osteoporose e câncer de mama, relacionados à saúde da mulher que subsidiaram o intercâmbio das informações entre estudantes e comunidade.

\section{MATERIAL E MÉTODOS}

Para o desenvolvimento desta pesquisa, considerando a questão norteadora: como avaliar a percepção dos participantes sobre um evento de extensão, comparando a avaliação feita pelas distintas comunidades. Realizou-se uma investigação quantitativa em três comunidades do Estado de Santa Catarina. O instrumento de pesquisa utilizado foi um questionário semiestruturado, com perguntas relacionadas à qualidade da palestra, dinâmicas utilizadas, meios de divulgação, entre outros. A pesquisa não apresentou quaisquer riscos aos sujeitos da pesquisa. Para garantir o sigilo os dados coletados foram registrados em um formulário de pesquisa sem identificação nominal dos participantes do estudo. O projeto ocorreu entre os meses de março a julho de 2013.

Quanto à natureza da pesquisa, classificou-se como uma pesquisa aplicada, pois “objetiva gerar conhecimentos para aplicação prática, dirigidos à solução de problemas específicos. Envolve verdades e interesses locais” (SILVA e MENEZES, 2005, p.20,).

Com relação aos objetivos, esta pesquisa pode ser considerada como exploratória e descritiva. Segundo Gil (2002, p.41), as pesquisas exploratórias têm "como objetivo 
Projeto de extensão - radiologia e comunidade: avaliação das atividades de promoção de saúde na comunidade

proporcionar maior familiaridade com o problema, com vistas a torná-lo mais explícito ou a constituir hipóteses. Pode-se dizer que estas pesquisas têm como objetivo principal o aprimoramento de ideias ou a descoberta de intuições". Ainda, "as pesquisas descritivas têm como objetivo primordial a descrição das características de determinada população ou fenômeno ou, então, o estabelecimento de relações entre variáveis" (GIL, 2002, p.42).

Essa ação de extensão utilizou como caminho metodológico a pesquisa ação, de natureza educativa, sendo que teve como finalidade desenvolver o aprimoramento educacional tanto dos docentes, como dos discentes e da comunidade. Investigação-ação é um termo utilizado para qualquer processo que segue um ciclo, onde se busca o aprimoramento da prática pela ação no, seu campo, e investigação a seu respeito (TRIPP, 2005).

Cumpriu-se uma carga horária de 40 horas, distribuídas em 20 horas de orientações semanais e mais 20 horas de atividades na comunidade. Participaram a professora coordenadora do projeto, uma professora colaboradora tecnóloga em radiologia, uma bolsista do Curso Superior de Radiologia, 14 estudantes da quinta fase do Curso Superior de Radiologia, 152 mulheres, 1 homem e uma criança. O projeto foi desenvolvido da seguinte forma: exposição das metas aos participantes e levantamento de sua percepção sobre o evento, levando em conta as palestras, debates e técnicas de dinâmica de grupo.

O projeto de extensão se propôs a realizar palestras que envolvem o processo de ensino-aprendizagem sobre algumas patologias, sendo uma delas o câncer de mama e a osteoporose que tem como principal característica, acometer principalmente as mulheres. Assim, o material didático era preparado de acordo com a comunidade escolhida, considerando a idade, as características socioeconômicas e culturais, abordando temas como a prevenção destas doenças, diagnóstico e fatores de risco.

Posteriormente às palestras, foram realizadas dinâmicas relacionadas aos alimentos e a sua quantidade de cálcio correspondente teatros e músicas também foram utilizados como forma de estímulo e promoção da sociabilidade entre os participantes.

No final do evento foi proposto o questionário elaborado pela bolsista juntamente com as professoras, aplicado aos participantes. O questionário teve como objetivo avaliar o evento a partir de itens como: andamento do evento e tipo de público atendido. Para análise do questionário, foram seguidos os seguintes parâmetros de exclusão: respostas incompletas e marcação de uma ou mais alternativas. 
Projeto de extensão - radiologia e comunidade: avaliação das atividades de promoção de saúde na comunidade

As perguntas foram estruturas de forma que as respostas fossem simples e facilitando o entendimento do público. Já para o responsável do grupo foi aplicado um questionário com informações adicionais a fim de conhecer e analisar o grupo de participantes.

Diferentes dos outros anos, em 2013, o projeto foi contemplado com o Programa de Extensão Universitária MEC/SESu - PROEXT 2014, que é um instrumento que abrange programas e projetos de extensão universitária, com ênfase na formação dos estudantes e na inclusão social nas suas mais diversas dimensões, visando aprofundar ações políticas que venham fortalecer a institucionalização da extensão no âmbito das Instituições Federais, Estaduais e Municipais de Ensino Superior. A partir desta data, as ações do Projeto Radiologia e Comunidade passaram a contar com uma ajuda de custo para a realização do evento.

Em 2013, o projeto contou com a participação de três diferentes grupos da turma de Radiologia-2011, dessa forma, cada um deles visitou sua própria comunidade que foi anteriormente escolhida, foram elas: comunidade do Santinho, comunidade da Costeira e no município de Garopaba, cidade próxima à Florianópolis.

\section{RESULTADOS E ANÁLISES}

Foram respondidos no total de 154 questionários pelo público geral, sendo que destes 117 foram considerados válidos, de acordo com os parâmetros de exclusão. A faixa etária dos participantes variou entre 10 a 89 anos, sendo somente uma pessoa do sexo masculino. A maioria das participantes, conforme demonstra a Ilustração 1, estavam na faixa etária entre 60 e 69anos, seguido do grupo entre 50-59 anos.

\section{Esta obra está licenciada sob uma Licença Creative Commons.}


Projeto de extensão - radiologia e comunidade: avaliação das atividades de promoção de saúde na comunidade

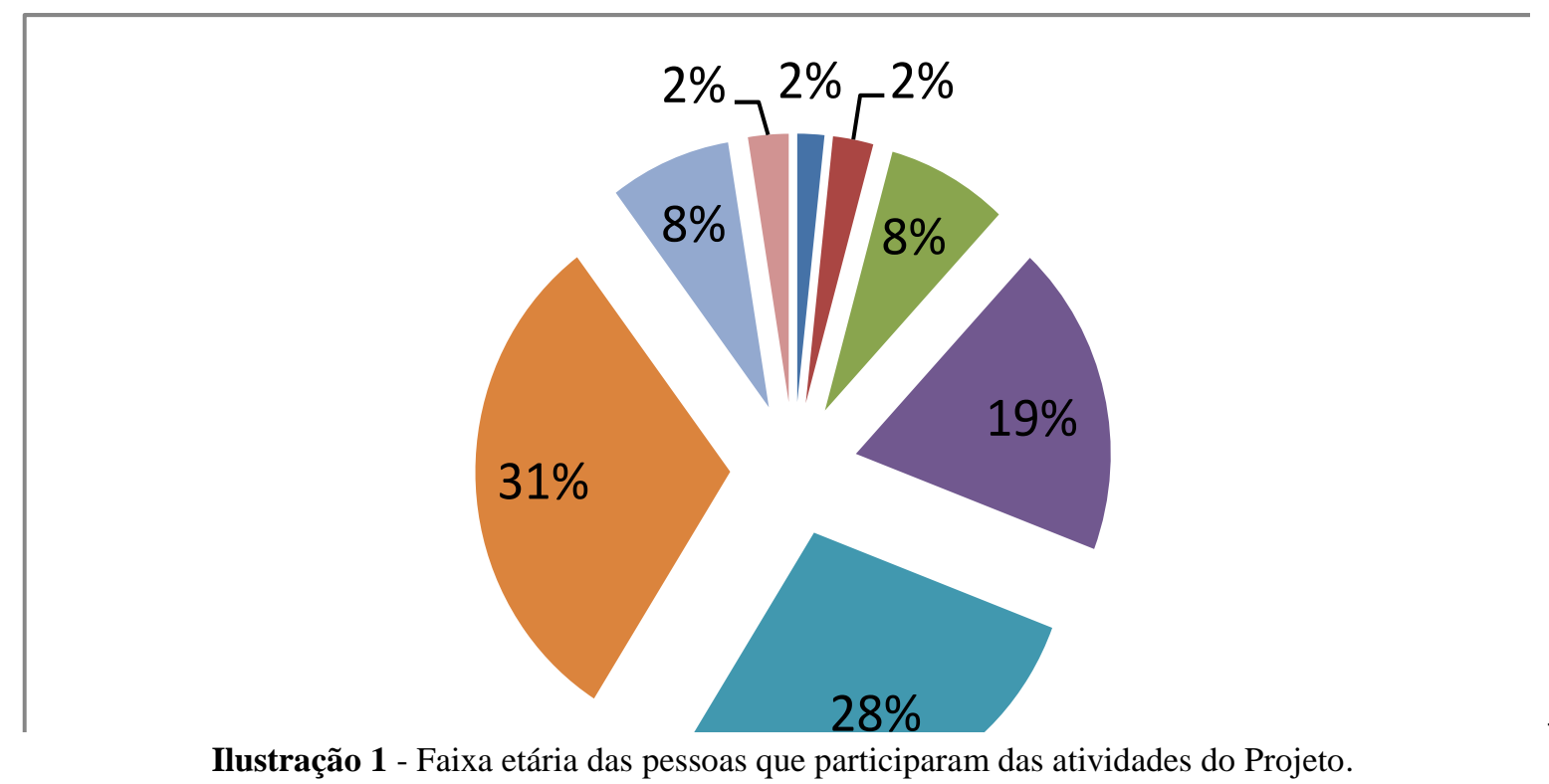

Fonte: Elaborado pelos autores.

Esse dado demonstra que a palestra atingiu o público alvo, já que ambas as patologias, osteoporose e câncer de mama, atingem principalmente as mulheres nesta faixa etária. Segundo Segura et al. (2007), o envelhecimento afeta o sistema ósseo, promovendo diminuição fisiológica da densidade mineral, podendo, inclusive, provocar uma doença sistêmica conhecida como osteoporose.

A osteoporose é a redução da massa óssea e deterioração da microarquitetura deste tecido, levando a uma fragilidade mecânica e predisposição a fraturas. Ainda relacionado a fatores de risco para o aparecimento do câncer de mama tem-se a vida reprodutiva da mulher, incluindo aspectos como menarca precoce, nuliparidade, idade da primeira gestação a termo acima dos 30 anos, anticoncepcionais orais, menopausa tardia e terapia de reposição hormonal, bem estabelecidos em relação ao desenvolvimento desta patologia. A idade continua sendo um dos mais importantes fatores de risco. As taxas de incidência aumentam rapidamente até os 50 anos e, posteriormente, esse aumento ocorre de forma mais lenta. Essa mudança no comportamento da taxa é conhecida na literatura como "Clemmesen's hook", e tem sido atribuída ao início da menopausa (BRASIL, 2009).

A respeito da avaliação do evento, os resultados obtidos quanto à avaliação do evento em geral estão apresentados nas Ilustrações 2, 3 e 4 .

\section{Esta obra está licenciada sob uma Licença Creative Commons.}


Projeto de extensão - radiologia e comunidade: avaliação das atividades de promoção de saúde na comunidade

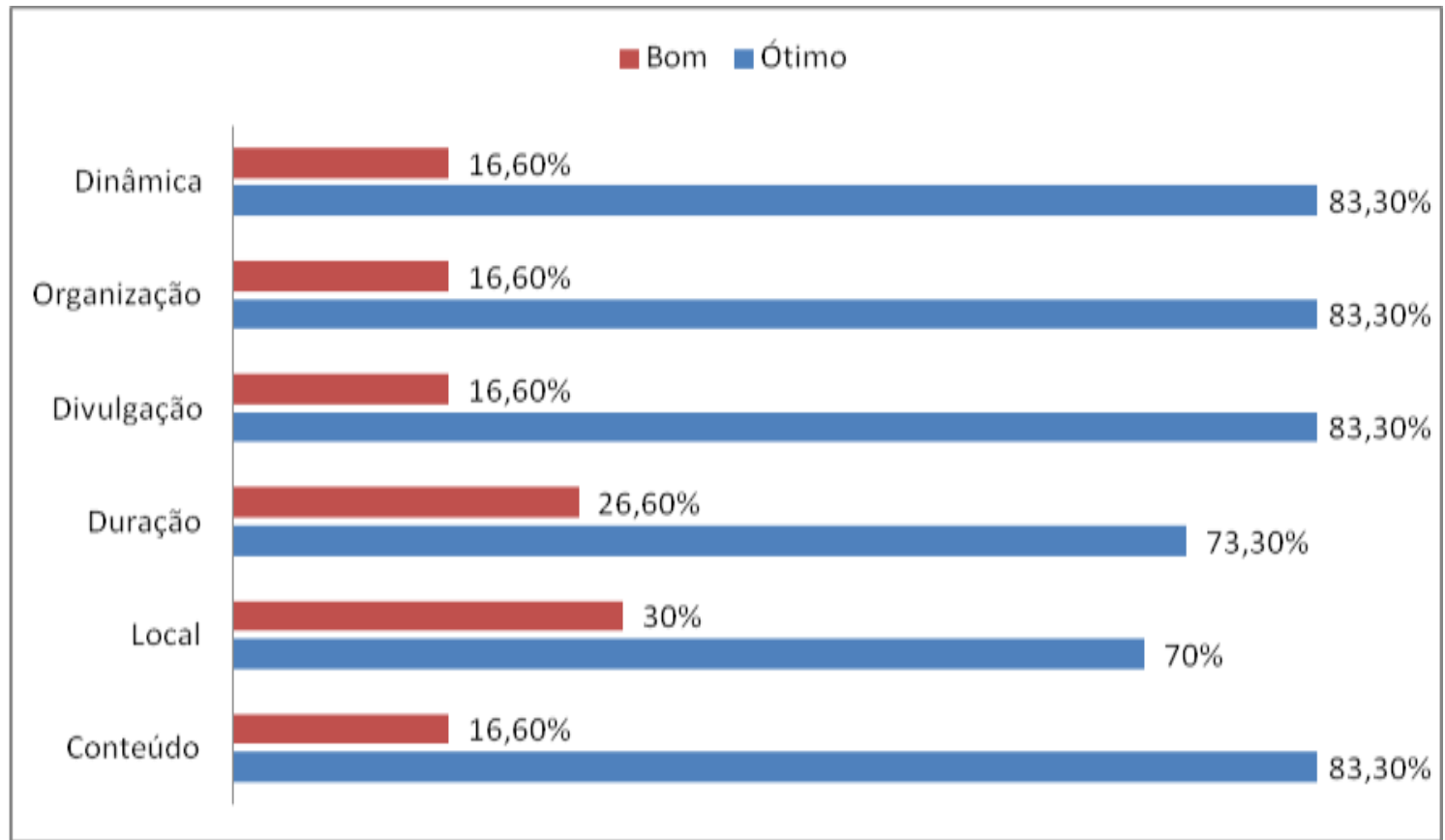

Ilustração 2 - Resposta às perguntas dirigidas a Comunidade do Santinho, março de 2013. Fonte: Elaborado pelos autores.

Mesmo com algumas diferenças pontuais observadas nas respostas das atividades, de maneira geral, as avaliações realizadas pelas comunidades do Santinho, Costeira e Garopaba foram consideradas de boa a ótima (Ilustraçãos 2, 3 e 4).

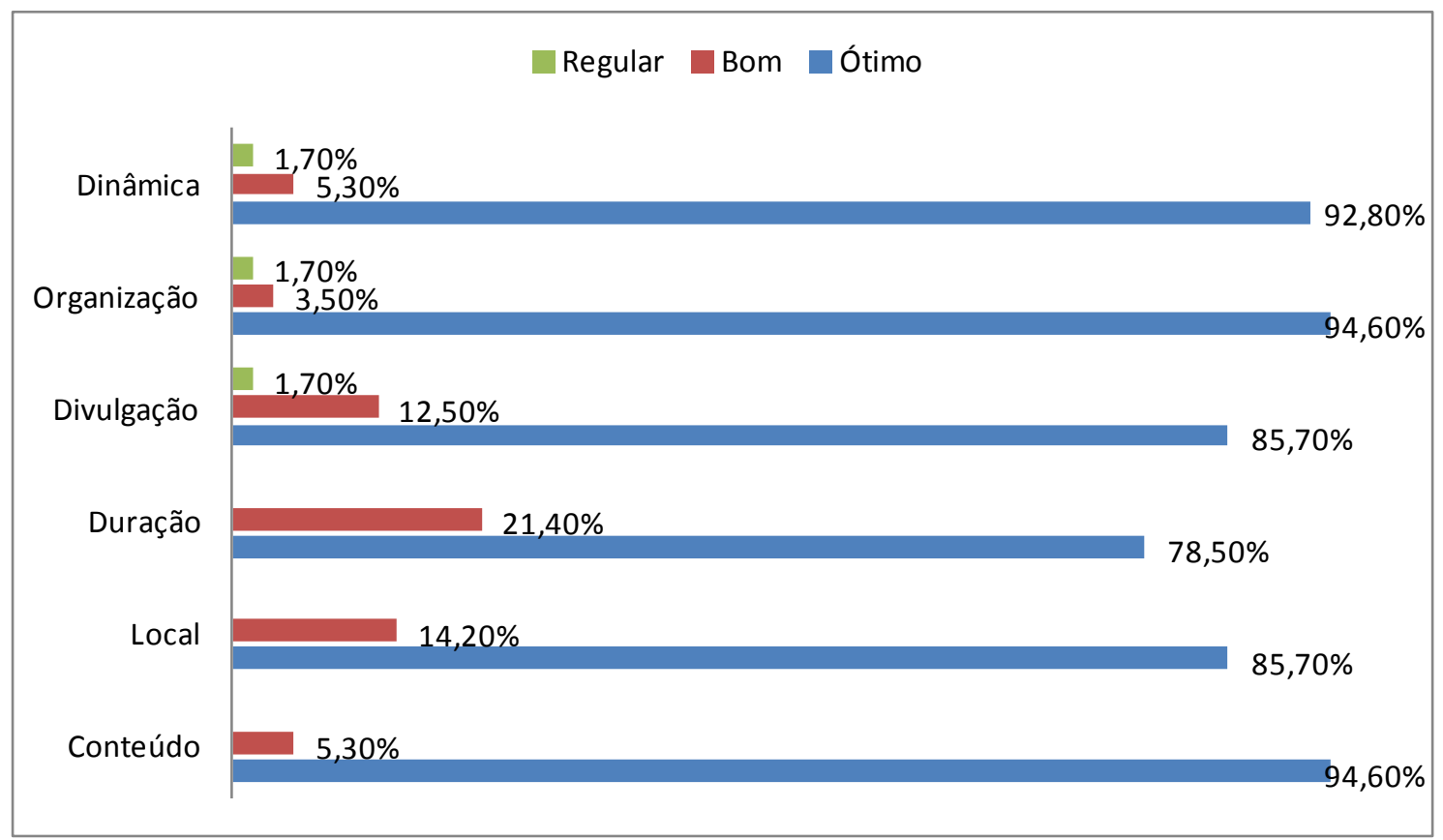

Ilustração 3 - Resposta às perguntas dirigidas a Comunidade da Costeira sobre o evento, março de 2013. Fonte: Elaborado pelos autores.

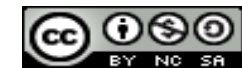

Esta obra está licenciada sob uma Licença Creative Commons. 
Projeto de extensão - radiologia e comunidade: avaliação das atividades de promoção de saúde na comunidade

Contudo, também é possível observar vários aspectos díspares entre respostas, o que pode ser explicadas partindo do pressuposto que em cada comunidade houve um grupo de estudantes diferente para desenvolver e apresentar a palestra, e, portanto, em cada um deles foi escolhida uma dinâmica organizacional distinta.

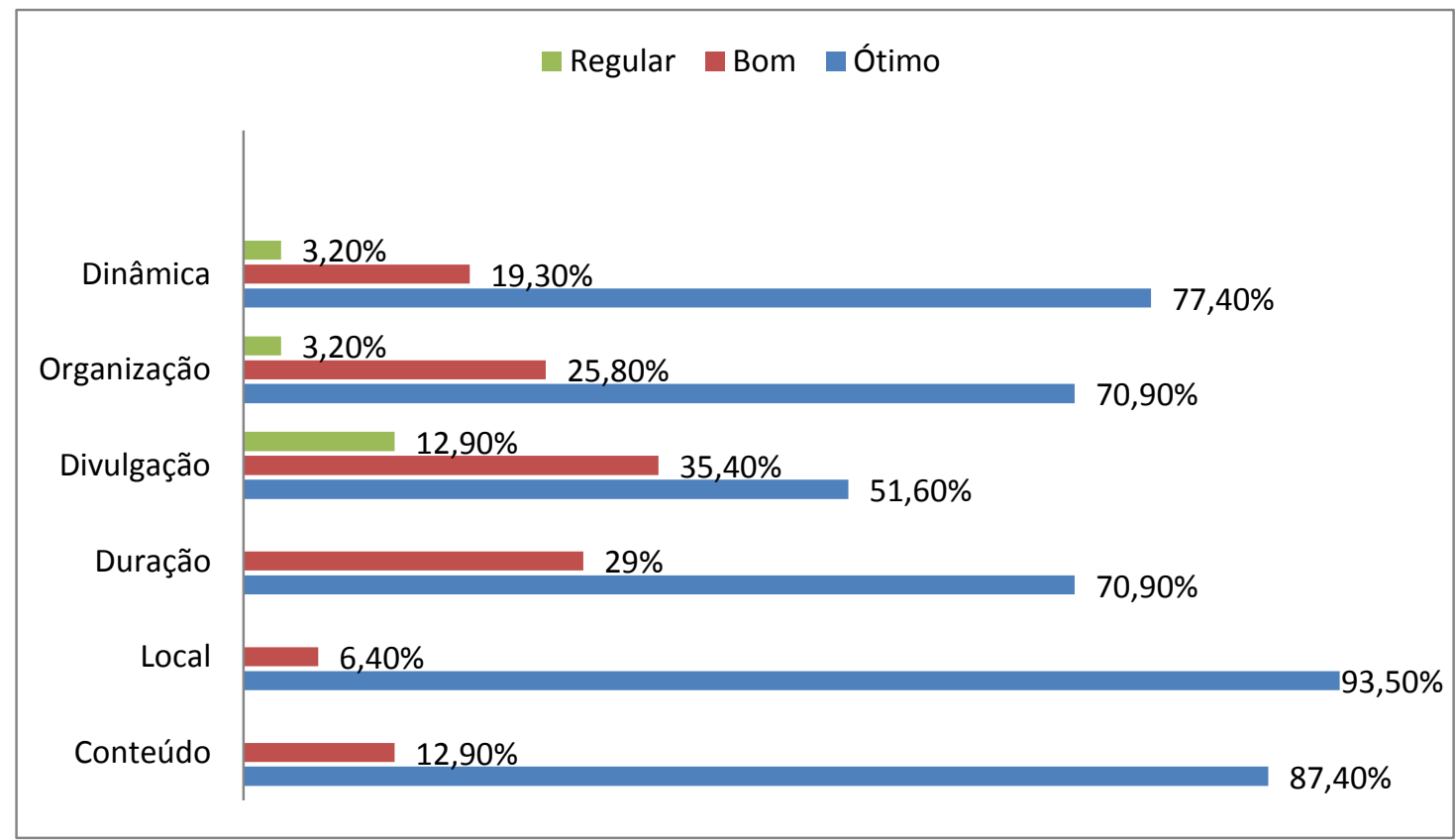

Ilustração 4 - Resposta às perguntas dirigidas a Comunidade de Garopaba sobre o evento, março de 2013. Fonte: Elaborado pelos autores.

A partir da análise dos questionários respondidos em todas as comunidades foi possível averiguar que o evento teve um alto nível de aceitação, desde o conteúdo repassado até a dinâmica em grupo para a fixação do conhecimento; já que todos os itens foram predominantemente considerados ótimos, havendo somente alguns requisitos de divulgação, organização e dinâmica que foram considerados regulares.

Nos questionários preenchidos pelos responsáveis da comunidade, percebeu-se que todos os grupos foram receptivos ao evento e julgaram de suma importância à discussão de temas relacionados à saúde da mulher. Mesmo que os grupos visitados realizem com frequência outras atividades que incluem a cuidados com a mulher e atividades de lazer.

Para alcançar os objetivos propostos pelo projeto, foi determinado um programa de educação em saúde, envolvendo palestras, folders e dinâmicas em grupo (Ilustraçãos 5). O principal objetivo era levar a informação de forma simples e clara para as comunidades sobre a prevenção, os métodos de diagnóstico e maneiras para evitar tais patologias. O único ponto

\section{(†) (-) Esta obra está licenciada sob uma Licença Creative Commons.}


Projeto de extensão - radiologia e comunidade: avaliação das atividades de promoção de saúde na comunidade

negativo identificado foi à dificuldade de agendamento de horário com as comunidades de forma que os estudantes pudessem comparecer.

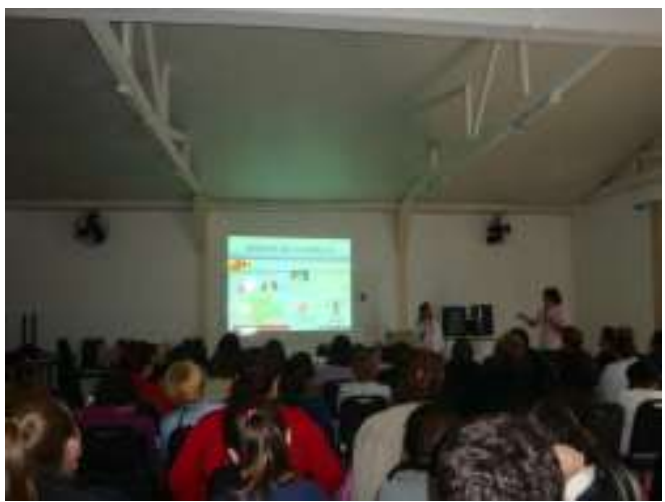

(a)

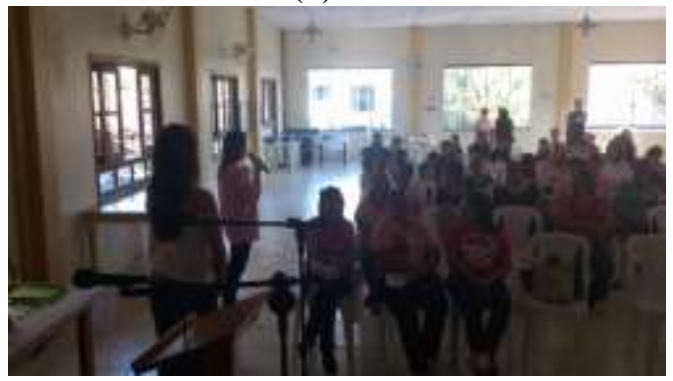

(c)

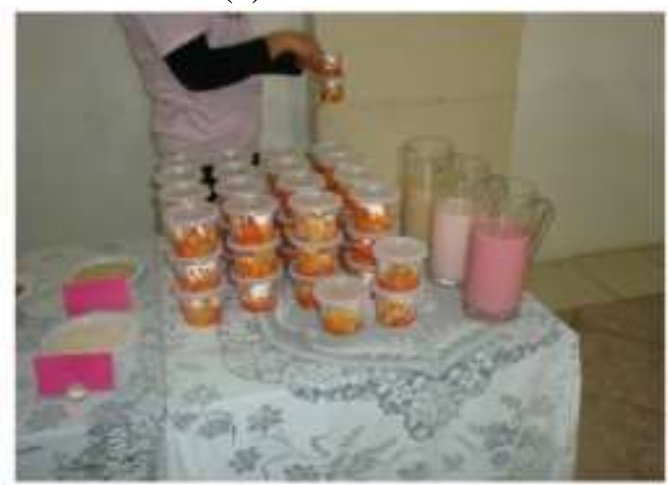

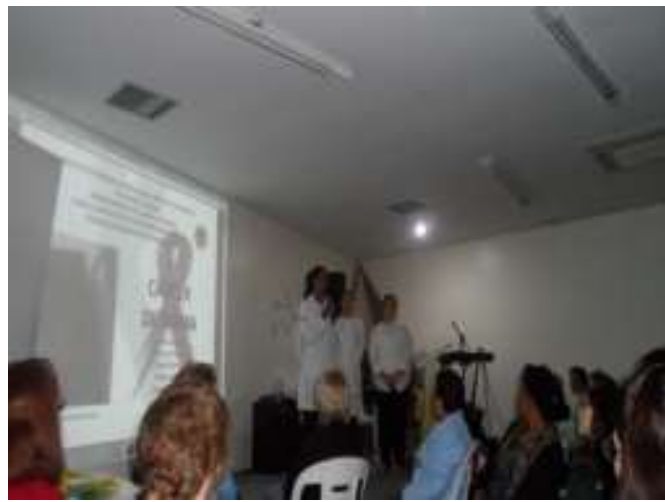

(b)

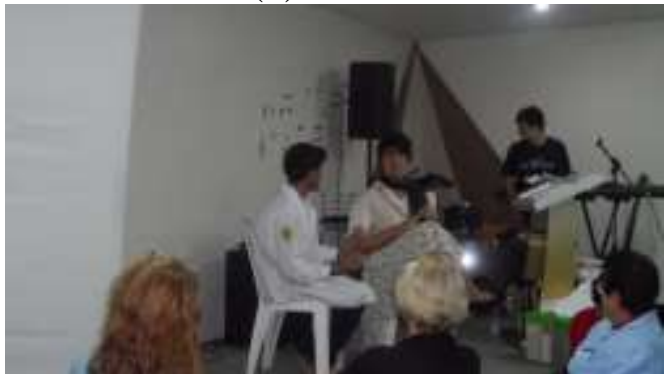

(d)

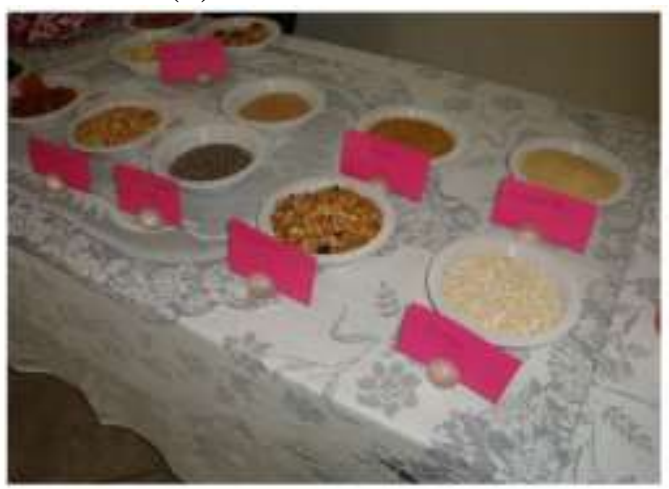

(e)

Ilustrações 5 -Palestras oferecidas nas três comunidades comtempladas no ano de 2013: (a) público na Comunidade do Santinho. (b) alunas do Curso Superior em Radiologia na Comunidade da Costeira. (c) dinâmica na Comunidade de Garopaba. (d) peça apresentada pelo teatro Boca de Siri do IF-SC (e) lanche oferecido no evento da Comunidade do Santinho, Florianópolis.

Fonte: Fotografado pelos autores.

O resultado refletiu que uma ação interdisciplinar e conjunta, ao considerar o diálogo via educação, saúde e cidadania, atende, tanto as necessidades da comunidade, quanto à 
Projeto de extensão - radiologia e comunidade: avaliação das atividades de promoção de saúde na comunidade

prática acadêmica. Nesse sentido, melhorar a qualidade de vida representa um desafio, tanto para os adultos e idosos atendidos como para os atores envolvidos nesse projeto.

\section{CONSIDERAÇÕES FINAIS}

A extensão universitária, diferentemente do ensino e da pesquisa, pouco foi regulamentada pelos órgãos de governo, tanto no que diz respeito à normalização de suas práticas, quanto do financiamento de suas atividades. "Este distanciamento imposto permitiu que ela se reorganizasse e pudesse se dedicar a construir uma relação mais próxima da sociedade" (CASTRO, 2004, p. 48).

Diante dos resultados obtidos, pode-se concluir que o Projeto "Radiologia e Comunidade" alcançou seus objetivos propostos, pois avaliou a percepção dos participantes sobre o evento de extensão universitária, comparando a avaliação feita pelas distintas comunidades. Foi possível constatar que a maioria dos participantes, 31\%, eram idosos e tinham entre 60 a 69 anos de idade. A dinâmica, organização, divulgação, o local e o conteúdo foram avaliados como ótimo na maioria dos casos, sendo que duas comunidades, Costeira e Garopaba avaliaram como regular os parâmetros relacionados a dinâmica, organização e divulgação, porém, esse número não chegou a 3,20\% dos casos.

Também foi verificado que esse projeto teve grande relevância tanto para a comunidade que pode conhecer e tirar suas dúvidas sobre as patologias relacionadas a saúde da mulher, quanto para os estudantes e professores que exerceram seus papéis de cidadãos conscientes ao compartilhar seus conhecimentos com a comunidades proporcionando que essa possa disfrutar de uma melhor qualidade de vida - ao conhecer formas tanto de prevenção quanto de tratamento.

Constata-se, com este artigo, que os projetos de extensão universitária são muito importantes para os estudantes das universidades brasileiras, docentes e para a comunidade atendida. Concluiu-se que a interação entre estudantes e comunidades é uma ótima forma para os estudantes transmitirem seus conhecimentos para a comunidade e aprenderem com ela formando uma espiral virtuosa de conhecimentos explícitos e tácitos.

\section{Esta obra está licenciada sob uma Licença Creative Commons.}


Projeto de extensão - radiologia e comunidade: avaliação das atividades de promoção de saúde na comunidade

\section{REFERÊNCIAS}

BRASIL. Ministério da Saúde. Instituto Nacional de Câncer. Estimativa 2010: incidência de câncer no Brasil / Instituto Nacional de Câncer. Rio de Janeiro: INCA; 2009.

CASTRO, Luciana M. Cerqueira. A universidade, a extensão universitária e a produção de conhecimentos emancipadores: ainda existem utopias realistas. 2004. 185 f. Tese (Doutor) - Universidade do Estado do Rio de Janeiro, Rio de Janeiro, 2004. Disponível em $<$ http://www.anped.org.br/reunioes/27/gt11/t1111.pdf $>$. Acesso em 01 de out de 2013.

GIL, A. C. Como elaborar projetos de pesquisa. São Paulo: Atlas, 2002.

KAMINSKI, L. Projeto integrado de Ação Universitária - PIAU: a atividade extensionista como forma de minimizar as desigualdades sociais. Mimeo. UNIOESTE, 2000 .

LOH, B.; GRABOSKY, J.C.; BASSKUK, N.L. Applying knowledge management in university research. In: (Orgs.). Governing and Managing Knowledge in Asia. Singapore, v.9, 2003. p. 221-248.

MARTINS, Josiane de Jesus; BARRA, Daniela Carvalho; SANTOS, Tatiana; HINKEL, Verenice; NASCIMENTO, Eliane; ALBUQUERQUE, Gelson; ERDMANN, Alacoque. Educação em saúde como suporte para a qualidade de vida de grupos de terceira idade. Revista Eletrônica de Enfermagem, Florianópolis, v. 09, n. 02, p. 443-456, ago., 2007.

MOREIRA, Bernadete; PELLIZZARO, Inês. Educação em Saúde: um programa de extensão universitária. Revista Textos \& Contextos, Porto Alegre, v. 8, n. 1, p. 156-171. jan./jun., 2009.

PAVIANI J. Disciplinaridade e Interdisciplinaridade. Revista de Estudos Criminais. v. 3, n. 12, p.59-85, 2003.

SALMI, J. Constructing knowledge societies: New challenges for tertiary education. Disponível em: $<$ http://siteresources.worldbank.org/INTAFRREGTOPTEIA/Resources/Constructing_Knowle dge_Societies.pdf $>$. Acesso em: 23 jul. 2013. 
Projeto de extensão - radiologia e comunidade: avaliação das atividades de promoção de saúde na comunidade

SEGURA, D. C. A.; NASCIMENTO, F. C.; PETROSKI, E. L.; KLEIN, D. FERMINO, D. Relação entre atividade física e osteoporose. Arq. Ciênc. Saúde Unipar, Umuarama, v. 11, n. 1, p. 51-50, jan./abr. 2007.

SILVA, E. L. da; MENEZES, E. M. Metodologia da pesquisa e elaboração de dissertação. 4. ed. rev. atual. Florianópolis: UFSC, 2005. 138p. Disponível em: <http://www.portaldeconhecimentos.org.br/index.php/por/content/view/full/10232>. Acesso: 01 out. 2011

TRIPP, David. Pesquisa-ação: uma introdução metodológica. Educação e Pesquisa, n. 3, v. 31, p. 443-466, set./dez., 2005. Disponível em: <http://www.scielo.br/pdf/ep/v31n3/a09v31n3.pdf >. Acesso em: 01 out. 2011 